\title{
Desafios e Novas Possibilidades de Uso de Learning Management Systems
}

\author{
João Pedro Dewes Guterres, Milene Selbach Silveira \\ PUCRS, Faculdade de Informática \\ Porto Alegre - RS - Brasil \\ joao.guterres@acad.pucrs.br, milene.silveiraepucrs.br
}

\begin{abstract}
Learning Management Systems (LMS) are important tools to learning support. In order to understand the state of art of these systems, a literature review was done, grounding a discussion about their challenges and possibilities. The paper highlights adjustments that need to be done to make these systems more open, personal, social, flexible, analytical and mobile. The idea is to support pedagogical and development teams, and researchers that work in this area and want to incorporate and construct new tools and methodologies in this field.
\end{abstract}

Resumo. Learning Management Systems (LMS) tem se destacado como uma importante ferramenta de apoio à aprendizagem. Para se entender o contexto atual destes sistemas, foi realizada uma revisão da literatura, a qual embasou também uma discussão sobre desafios e novas possibilidades de uso destes sistemas. Foram identificadas adaptações que necessitam ser feitas para tornar este tipo de sistema mais aberto, pessoal, social, flexível, analítico e móvel. A ideia é que as discussões abordadas apoiem as equipes pedagógicas, de desenvolvimento e pesquisadores que atuam nesta área na incorporação e construção de novas ferramentas e metodologias neste campo.

\section{Introdução}

A inserção de novas tecnologias no sistema educacional tem estimulado as áreas acadêmica e empresarial a criarem soluções para esse segmento. Nesse contexto, a utilização destas soluções tem levantado questões relevantes, tanto para o aprofundamento científico, quanto para a gestão pedagógica desses recursos. Este movimento faz surgir uma série de perspectivas e enfoques diferenciados, dentre os quais a utilização de LMS (Learning Management Systems) se destaca como uma importante ferramenta de apoio à aprendizagem, sendo ela utilizada em aulas a distancia ou como apoio à aula presencial [Barros e Carvalho 2011; Ninoriya et al. 2011].

Fundamentados na utilização de TIC (Tecnologias de Informação e Comunicação) na educação, muitos sistemas de LMS estão sendo incorporados na sistemática de ensino de disciplinas, cursos e treinamentos. O desafio a partir dessa premissa está em explorar esses recursos de maneira eficaz, melhorando e criando novas possibilidades para a gestão da aula, sendo ela no meio escolar, acadêmico e/ou empresarial. 


\section{CBIE-LACLO 2015}

Anais do XXVI Simpósio Brasileiro de Informática na Educação (SBIE 2015)

Pesquisas recentes revelam que, na modalidade EAD (Educação a Distância), observou-se que os estudantes que tinham os maiores índices de acesso a ferramentas LMS, coincidentemente, obtiveram as maiores pontuações no exercício avaliativo [Silva et al. 2014; Detoni e Araujo 2014]. Entretanto, com estudantes presenciais, evidenciouse que estas ferramentas estão sendo subutilizadas, não tendo tanta influência no desempenho dos que as utilizaram como apoio a aula presencial [Santana et al. 2014].

Novas pesquisas buscam, então, tanto analisar a importância desses recursos quanto explorar novos modos de utilizá-los de forma a aprimorar a qualidade do processo de ensino e de aprendizagem. Considera-se que a introdução de novos meios tecnológicos e de novas metodologias de ensino irão produzir efeitos positivos na aprendizagem, porque esses meios poderão modificar e melhorar o modo como os professores ensinam e também como os alunos aprendem [Leite 2014].

Sendo assim, conhecer os recursos disponíveis e as novas ferramentas utilizadas nos LMS apresenta-se como uma necessidade para a construção de uma base teórica e científica que permita subsidiar trabalhos futuros neste segmento. Nesse sentido, a literatura apresenta projetos promissores utilizando novas técnicas e ferramentas que apoiam a gestão de aprendizagem, sendo estas destinadas tanto a aulas a distancia quanto ao apoio à aula presencial.

A partir de uma revisão da literatura, considerando trabalhos relacionados à área de gestão de aprendizagem e gestão de conteúdos de aprendizagem, esta pesquisa aprofunda trabalhos recentes que abordam ferramentas e modelos de aprendizagem diferentes dos LMS tradicionais. A partir desses trabalhos, é feita uma discussão, buscando contribuir tanto na criação de ferramentas para serem incorporadas nos LMS quanto propor novas possibilidades de uso destes sistemas.

A ideia é que as discussões abordadas apoiem as equipes pedagógicas, de desenvolvimento e pesquisadores que atuam nesta área na incorporação e construção de novas ferramentas e metodologias em seus sistemas LMS.

Este trabalho está organizado em cinco seções: a seção 2 apresenta conceitos básicos dos sistemas LMS e exibe os principais softwares que implementam esses sistemas; a seção 3 apresenta trabalhos relacionados que apresentam novas ferramentas utilizadas LMS; a seção 4 discute esses trabalhos, investigando problemas e soluções encontradas por eles e propondo novas possibilidades de uso nos LMS; e a seção 5 apresenta as conclusões deste trabalho, seguidas das referências utilizadas para sua elaboração.

\section{Conceitos Básicos}

Os LMS, segundo Ellis e Calvo (2007), são sistemas de gestão de aprendizagem projetados para apoiar a aprendizagem do aluno. Seu objetivo principal é prover uma infraestrutura para organizar e apoiar o aprendizado, sendo ela no apoio à aula presencial ou servindo de base para aulas e cursos EAD, possuindo ferramentas para exibição de conteúdos, avaliações, monitoramento e comunicação [Tarouco et al. 2010].

Nesse tipo de sistema é possível gerenciar usuários e suas permissões, tendo cada usuário uma visão diferente do sistema para interagir. $\mathrm{Na}$ versão tradicional do LMS, os professores disponibilizam materiais educacionais e canais de discussões em 


\section{CBIE-LACLO 2015}

Anais do XXVI Simpósio Brasileiro de Informática na Educação (SBIE 2015)

suas disciplinas e os alunos tem acesso ao repositório de recursos educacionais disponibilizados, além da possibilidade de interagir com professores e colegas por meio de ferramentas como chat, fórum de discussões, wikis e blogs [Wang e Chen 2009]. Segundo Barros e Carvalho (2011), LMS oferecerem características de controle e gerenciamento inexistentes em outras interfaces da web, possuindo uma dinâmica própria para atender ao fazer pedagógico, o qual é orientado no sentido de que se estabelecem metas para o aluno atingir.

Como exemplos de LMS, podem-se citar alguns dos sistemas mais utilizados no mundo em 2014 [Capterra 2014]: Moodle, Edmodo, Blackboard, Canvas (Instructure) e Desire2Learn (D2L). Realizando uma análise comparativa entre eles, destaca-se que a maioria possui características dos LMS tradicionais, com somente os professores responsáveis pela inserção de materiais expositivos. Outra característica está na ausência de ferramentas que permitam a utilização de novos modelos de aprendizagem, como a aprendizagem baseada em problemas, em projetos, etc.

A interdisciplinaridade entre cursos e disciplinas que pode ser vista no BlackBoard ${ }^{1}$, por exemplo, ainda é uma barreira imposta por quase todos os LMS tradicionais, não permitindo a criação de grupos por alunos.

A interface dos LMS ainda não é considerada intuitiva se comparada com as redes sociais. Nesse contexto, o Edmodo ${ }^{2}$ se destaca como uma rede social educativa, incorporando recursos de colaboração na criação de arquivos. Mas, se por um lado este LMS tem recursos comparados a uma rede social, ainda faltam a ele recursos de um LMS tradicional, que possui ferramentas mais robustas para realizar o acompanhamento do aluno e integração com sistemas legados da instituição de ensino.

$\mathrm{Na}$ seção seguinte são apresentados projetos que estão incorporando novas ferramentas e métodos para aprendizagem em LMS.

\section{Trabalhos Relacionados}

Com o objetivo de analisar novas possibilidades de uso dos LMS, foi realizada uma pesquisa em projetos recentes que utilizaram recursos para proporcionar uma melhor aprendizagem aos alunos. Estes trabalhos são, a seguir, apresentados de acordo com suas características e enfoques.

\subsection{Social LMS}

Para aproximar ambientes LMS de redes sociais, iniciaram-se pesquisas e projetos no campo denominado Social LMS [Papić e Bešter 2012]. Nesse contexto, os sistemas se dividem em dois tipos: os que permitem simples integrações com redes sociais; e os que oferecem uma coleção de ferramentas utilizadas nas principais redes sociais, de modo a se tornar um SNE (Social Learning Environment).

O Topyx ${ }^{3}$ é um LMS que faz parte do primeiro tipo, ao permitir a integração com redes sociais como Facebook, Twitter e Linkedin. Nesse contexto, o estudante pode

\footnotetext{
${ }^{1}$ www.blackboard.com

2 www.edmodo.com

${ }^{3}$ www.interactyx.com
} 
estar conectado nessas redes sociais sem sair no ambiente de aprendizagem. Já Braz et al. (2011) propõem um mecanismo pelo qual é possível ter acesso aos conteúdos do Moodle pelo Facebook, de forma de motivar os alunos a serem mais participativos e engajados nas tarefas propostas no LMS.

Por outro lado, o E-CHO ${ }^{4}$ faz parte do segundo tipo: o sistema possui ferramentas sociais de colaboração, tais como criação de grupos e chats, possibilitando a criação de posts e compartilhamento de arquivos [Papić e Bešter 2012]. O E-CHO se difere dos LMS tradicionais ao possibilitar que a criação de grupos seja feita pelos próprios alunos, favorecendo a criação de grupos multidisciplinares, onde eles se organizam de acordo com seus interesses, independentes de semestre ou curso matriculado. Nesta mesma linha encontra-se, também, o Edu 2.0 [Ivanova e Popova 2009]. Seus autores o denominaram como LMS 2.0, por possuir ferramentas baseadas em tecnologias da web 2.0 de forma a proporcionar tanto aprendizagem formal quanto informal, apoiando um processo de aprendizado dinâmico e aberto, mais parecido com as funcionalidades de interação encontradas nas redes sociais.

No contexto do LMS sendo uma ferramenta mais social e menos rígidos aos alunos, ambos os tipos de LMS adotados podem ser utilizados para criar novos ambientes de aprendizagem. Esses ambientes, se comparados com as redes sociais, podem ser mais flexíveis aos alunos, permitindo a livre organização de diversos grupos de interesse, podendo ser apoiados por professores em um ambiente controlado.

\subsection{LMS Controlando Laboratórios Remotos em Tempo Real}

Uma das características principais dos LMS é a capacidade de reunir estudantes geograficamente distantes. Nesse contexto, Logar et al. (2011), além de integrarem alunos geograficamente distantes, integraram também um laboratório real onde os alunos tinham possibilidades de interagir com esse laboratório utilizando ferramentas do LMS em conjunto com outros softwares.

Utilizando o LMS E-CHO de forma adaptada a este experimento, foi possível controlar múltiplas variáveis para realizar os experimentos em busca da melhor performance. Esse projeto piloto foi amplamente aceito pelos alunos, de forma a estimular o seu estudo online e possuir novas possibilidades realizar exercícios em laboratório, além de propiciar cooperação entre estudantes de um grupo e a liberdade de organizar seus estudos.

Já Krneta et al. (2014) implementaram um ambiente remoto controlado pelo Moodle, mas com uma a abordagem de aprendizagem diferente, levando os alunos aprendem de forma exploratória, discutindo individualmente seus resultados com seus colegas. Nesse ambiente os alunos puderam ter acesso a esse laboratório, manipulando um equipamento de alto custo com o objetivo de preencher a lacuna existente entre formalismo matemático e a teoria do processamento de sinais.

Analisando ambos os contextos, observa-se que a utilização de um LMS para controle de variáveis em laboratórios remotos propicia ao aluno a capacidade de interligar a teoria com a prática. Essa capacidade de verificar o que está acontecendo no

\footnotetext{
${ }^{4}$ www.e-cho.org/
} 
ambiente real pode ser utilizada em diferentes contextos, se tornando um importante recurso para experimentação e fixação de conteúdos.

\subsection{Aprendizagem Baseada em Competição}

Para estimular o engajamento e a competição entre alunos, foi proposto um novo modelo de utilização de LMS combinando a Aprendizagem Baseada em Competição com a Aprendizagem Baseada em Projetos [Issa et al 2014]. Esse modelo foi criado a fim de ser uma solução alternativa para deficiências associadas a práticas tradicionais de aprendizagem, com o principal objetivo de criar habilidades de competição nos alunos. Em uma ferramenta "Workshop", os alunos avaliam aos pares outros grupos, comentam e julgam os trabalhos dos colegas, realizando feedbacks e classificando os outros projetos. Segundo os autores, a aplicação da competição entre os alunos atuando em grupo estimula habilidades como: autonomia, responsabilidade, automotivação, autoestima, competividade, criatividade, trabalho em equipe, habilidades de empreendedorismo e capacidades cognitivas e afetivas.

Ainda no contexto da competição, Swacha e Baszuro (2014) propuseram um modelo de LMS contendo mecanismos de gamificação, podendo distribuir materiais de aprendizagem a alunos, analisar seu progresso e permitir uma livre comunicação entre eles, individualmente ou em grupos.

É possível observar que ambos os trabalhos buscaram incorporar novos modelos de aprendizagem no LMS, inserindo práticas de competição e trabalho em equipe. Nesse contexto além das habilidades de competição, ambas as propostas buscam aumentar a colaboração entre alunos, seja para avaliar o trabalho do colega de forma construtiva ou para agir como um tutor, auxiliando colegas de competição.

\subsection{LMS Intersemestral e Interdisciplinar}

Uma das principais lacunas dos LMS tradicionais está na rigidez imposta ao limite de tempo que o curso está disponível e a impossibilidade de interligar disciplinas. Nesse contexto, Mott e Wiley (2009) elaboraram um modelo híbrido de OLN (Open Learning Network), composto por um LMS e um PLE (Personal Learning Environment).

Segundo Mott e Wiley (2009) há uma ruptura social e cognitiva entre o nível de interação do aluno com os LMS. Essa ruptura ocorre ao final de cada semestre universitário, quando o prazo de uma disciplina expira e o aluno não tem mais acesso a ela. Nesse panorama é proposta a intersemestralidade de disciplinas, a fim de propiciar um panorama mais amplo de aprendizagem, e também a interdisciplinaridade. As disciplinas, principalmente de um mesmo curso, são interligadas entre si, sendo, muitas vezes, pré-requisito de outra. Esse movimento intersemestral visa ampliar os conhecimentos do aluno, criando grupos multidisciplinares de alunos interessados em uma determinada área de conhecimento.

Já Park e Mills (2014), para empregar a aprendizagem interdisciplinar, utilizaram o BlackBoard com um módulo interdisciplinar entre diferentes disciplinas, o qual apresentou um grande nível de satisfação por parte dos alunos. Neste projeto, para criar comunicação, interatividade, colaboração e condições para aprendizagem construtivista, foram utilizados como recursos de interdisciplinaridade o Podcast, o Grupo Virtual, e o Quadro de Discussões. 


\section{CBIE-LACLO 2015}

Anais do XXVI Simpósio Brasileiro de Informática na Educação (SBIE 2015)

Pelos resultados dessas diferentes abordagens de aprendizagem, pode-se perceber a preferência por uma modalidade autodirigida e colaborativa, tendo os professores como tutores para eventuais intervenções.

\section{Discussão}

Com a intensa mudança de tecnologias e inclusão de dispositivos móveis ao dia a dia dos estudantes, os sistemas LMS tradicionais necessitam se adaptar a esse novo panorama para satisfazer as atuais necessidades dos alunos. Nesse contexto, Stone e Zheng (2014) destacam que a nova geração de LMS necessita ser mais aberta, pessoal, social, flexível, analítica e móvel. Considerando sua abrangência e o alinhamento com os trabalhos aqui apresentados, será utilizada esta mesma denominação para discutir as perspectivas futuras desta área.

\subsection{LMS Mais Aberto}

Segundo Mott (2010), os LMS tradicionais impedem o ensino e aprendizagem inovadores em três sentidos. O primeiro é a interrupção da continuidade e fluxo do processo de aprendizagem de uma disciplina, que possuem validade de tempo e expiram ao final do semestre, fazendo o aluno perder acesso a seus conteúdos. O segundo é a centralização da administração do curso no professor, não possibilitando muitas vezes a um aluno criar discussões, incluir arquivos e formar grupos. O terceiro é a impossibilidade de contato com alunos e disciplinas de outros cursos, tornando os alunos restritos apenas a seus cursos de graduação.

Nesse contexto, é possível observar que os trabalhos no campo Social LMS estão desenvolvendo LMS com mais liberdade ao aluno, onde ele pode criar grupos de alunos que quebram as barreiras da interdisciplinaridade e intersemestralidade. A proposta de Mott e Wiley (2009), ao elaboraram um novo modelo híbrido de OLN, também vem ao encontro dessa diretriz, tornando o aluno um agente ativo e participativo em suas disciplinas (em curso ou já cursadas).

Além disto, ao possibilitar as experimentações remotas e ambientes baseados em competição, se permite uma maior cooperação entre alunos. Ao invés de utilizar o mesmo experimento semestre após semestre com novos alunos, é possível utilizar experiências anteriores como exemplo de boas práticas e podendo contar com a experiência de alunos passados.

\subsection{LMS Mais Pessoal}

Segundo o paradigma PLE (personal learning environment) [Harmelen 2006], para proporcionar um LMS mais pessoal ao aluno, ele deverá incorporar um modelo pelo qual o aluno poderá gerenciar sua própria aprendizagem.

Com a proximidade entre os LMS e as redes sociais, o aluno terá mais liberdade para criar grupos e, com isso, ficar mais tempo no LMS. Somando a essa característica outras possibilidades, como a capacidade de criar e armazenar seus próprios repositórios de conteúdos de aprendizagem, o aluno poderá centralizar seu ambiente de estudo no LMS. Essa centralização do ambiente de estudos no LMS aliada aos recursos robustos do LMS de rastrear suas ações possibilitará as instituições um maior entendimento das dificuldades dos alunos, podendo atuar mais ativamente em sua aprendizagem. 


\section{CBIE-LACLO 2015}

Anais do XXVI Simpósio Brasileiro de Informática na Educação (SBIE 2015)

Como foi observado nos trabalhos apresentados, algumas dificuldades de aprendizagem poderão também ser resolvidas pelos próprios colegas, não apenas pelo professor. Se observarmos o LMS mais pessoal, combinando intersemestralidade e interdisciplinaridade, pode-se obter uma rede de aprendizagem muito ampla, onde a colaboração e cooperação entre alunos se torne uma prática mais ativa.

\subsection{LMS Mais Social}

As redes sociais, em virtude de suas características socializantes e sua capacidade de manter seus usuários conectados, têm sido utilizadas como ambientes para apoiar a educação [Junior et al. 2014]. Entretanto elas não possuem ferramentas robustas para controle de acesso e avaliação e participação dos alunos, sendo capaz - em sua maioria - apenas de contabilizar posts e visualizações. Nesse contexto, acredita-se que o caminho a ser tomado deve ser o contrário. Ao invés de tentar trabalhar em redes sociais transformando-as em LMS, deve-se tentar tornar o LMS mais social.

Com uma capacidade de socialização maior, os LMS sociais possuem características atrativas presentes nas redes sociais, tais como grupos orientados por objetivos, não apenas por disciplinas. Ao permitir essa organização, será possível mapear estudos, colaborar e compartilhar conhecimentos e experiências. A socialização do LMS contribui para o desenvolvimento de outras habilidades, tais como a aspectos sociais, comunicativos e competitivos.

Segundo Du et al. (2009), o senso de comunidade pode ajudar os alunos a serem mais engajados em uma classe, aumentando a retenção e resultados de aprendizagem. Tomando como exemplo o uso das redes sociais para a aprendizagem, há a possibilidade de mudar a estrutura de aprendizagem, propondo uma rede de relacionamentos com a comunicação entre pessoas baseada em posts. A teoria do Conectivismo [Siemens 2005] tem como princípio que a aprendizagem e o conhecimento são derivados de diversas opiniões, que são, então, conectadas. Podem-se comparar indivíduos a nodos que possuem conexões em rede, e o roteamento de informações entre esses nodos conecta ideias e conceitos que antes não eram possíveis. Ao transformar o LMS em um ambiente social, essas conexões são ampliadas, criando novas possibilidades de aprender com diferentes visões.

\subsection{LMS Mais Flexível}

A adaptação dos LMS para serem mais flexíveis vem ao encontro das necessidades de formar alunos com habilidades tanto cognitivas quanto não cognitivas. Para conquistar isso, os LMS precisam implementar soluções criativas para apoiar metodologias de ensino relacionadas tanto à aprendizagem informal, desenvolvendo habilidades como resolver problemas quanto à aprendizagem não-formal, desenvolvendo habilidades como trabalho em grupo como complemento da aprendizagem formal [Stone e Zheng, 2014].

Para chegar nessa solução, a estrutura do LMS precisa apoiar alternativas de aprendizagem como as baseadas em competição, projetos, problemas, etc. Essas podem ser utilizadas para aumentar o engajamento dos alunos, incentivando o desenvolvimento de habilidades tradicionalmente não cobertas pela aprendizagem formal. 


\section{CBIE-LACLO 2015}

Anais do XXVI Simpósio Brasileiro de Informática na Educação (SBIE 2015)

\subsection{LMS Mais Analítico}

Segundo Silva et al. (2014) e Detoni e Araujo (2014) há evidencias que os estudantes na modalidade EAD que tem os maiores índices de acesso nas ferramentas LMS obtêm melhores resultados avaliativos. Para confirmar essa evidencia, Santana et al. (2014) realizaram experimentos que confirmam a viabilidade de se predizer o desempenho dos estudantes utilizando técnicas de mineração de dados.

A extração de informações relevantes no ambiente acadêmico é um assunto muito abordado recentemente, atuando na área de Visual Analytics e Business Intelligence. Nesse contexto, os dados do LMS são utilizados para fornecer padrões dos estudantes, de forma de apoiar a tomada de decisão por parte de professores e gestores.

Ao tornar o LMS mais aberto, flexível, social e pessoal, será possível que o próprio aluno tenha uma maior autonomia para controlar seus estudos. Tendo o aluno acesso a informações relevantes sobre seu desempenho, ele pode tomar iniciativas de modo a intensificar seus estudos com o objetivo de melhorar seu desempenho.

\subsection{LMS Mais Móvel}

Tendo em vista que a maioria dos LMS tradicionais foi criada antes da geração dos dispositivos móveis, está aberta uma lacuna para explorar sua aplicação nesses dispositivos, com o intuito não somente de adequar o LMS às novas tecnologias, mas também como forma oferecer subsídios motivacionais aos alunos para a realização de atividades bem como explorar novos padrões de interação.

Em Sun (2014), são utilizados dispositivos móveis durante a aula para a realização de questionários por meio da estratégia de Just-in-Time teaching. Os resultados dessa pesquisa comprovaram um aumento do nível de atenção e relaxamento do aluno ao utilizar um dispositivo móvel para responder questionários durante a aula. Nesse contexto, a utilização de dispositivos móveis é um recurso desinibidor do aluno; num contexto maior ele pode ser utilizado para proporcionar novas formas de interação e de execução de tarefas por parte do usuário.

Ao proporcionar o LMS mais próximo do aluno através de aplicativos em dispositivos móveis, acredita-se ser possível obter um maior engajamento dos alunos.

\section{Considerações Finais}

Com o avanço das tecnologias, soluções inovadoras utilizando LMS têm sido desenvolvidas para apoiar a aprendizagem, sendo elas utilizadas em aulas a distancia ou como apoio à aula presencial. Foram apresentados, nesse artigo, conceitos básicos do LMS e suas principais características, além de trabalhos recentes com foco na apresentação de novas possibilidades de seu uso para ensino no meio acadêmico e/ou empresarial. Esses trabalhos foram agrupados de acordo com suas características e enfoques, de modo a serem explorados na discussão sobre as características da nova geração de LMS.

Observou-se que a utilização de novos recursos nesta área pode ser ainda mais abrangente se considerarmos um LMS mais aberto, pessoal, social, flexível, analítico e móvel, que proporcione formas alternativas de interação, busca de conhecimento e desenvolvimento de novas habilidades pelos alunos. Espera-se que as discussões 
realizadas possam auxiliar, aos interessados nesta área, apresentando-lhes alguns dos desafios atuais e possibilidades para pesquisas futuras no que se refere a novas perspectivas para aprendizagem utilizando LMS.

\section{Referências Bibliográficas}

Barros, M.G.; Carvalho, A.B.G. (2011) "As concepções de interatividade nos ambientes virtuais de aprendizagem”. In: Campina Grande: EDUEPB, 2011.

Braz, L.M.; Serrão, T.S.; Pinto, S.C.C.; Clunie, G. (2011) "Um Mecanismo para a Integração entre o LMS Moodle e o Site de Redes Sociais Facebook". In: XXII Simpósio Brasileiro de Informática na Educação (SBIE 2011), p. 904-913.

Capterra. (2014) "Best LMS (Learning Management System) Software 2015 | Reviews of the Most Popular Systems". In: <http://www.capterra.com/learning-managementsystem-software $/$ ?utf8=\%E2\%9C $\% 93 \& v=4 \#$ infographic $>$. Acesso em: 30/04/2015.

Du, H.; Rosson, M.B.; Carroll, J.M.; Ganoe, C. (2009) "I felt like a contributing member of the class: Increasing class participation with Class Commons". In: ACM 2009 International Conference on Supporting Group Work, p. 233-242.

Ellis, R.A.; Calvo, R.A. (2007) "Minimum Indicators to Assure Quality of LMSsupported Blended Learning. Educational Technology \& Society". Journal of Education Technology \& Society, vol. 10-2, 2007, pp 60-70.

Harmelen, M.V. (2006) "Personal Learning Environments". In: VI International Conference on Advanced Learning Technologies (ICALT 2006), p. 815-816.

Issa, G.; Hussain, S.M.; Al-BahadiliI, H. (2014) "Competition-Based Learning: A Model for the Integration of Competitions with Project-Based Learning using Open Source LMS". X International Journal of Information and Communication Technology Education (IJICTE 2014), n. 1, p. 1-13.

Ivanova, M.; and Popova, A. (2009). "An exploration of formal and informal learning flows in $\operatorname{lms}$ 2.0: Case study edu 2.0". In: Web Intelligence and Intelligent Agent Technologies (WI-IAT 2009), p. 227-230.

Junior, N.N.; Pimentel, E.P.; Dotta, S.C. (2014) "Gerenciamento da Participação e Desempenho de Estudantes em Atividades Educacionais no Facebook". In: XXV Simpósio Brasileiro de Informática na Educação (SBIE 2014), p. 574-583.

Krneta, R.; Brkovic, M.; Damnjanovic, D.; Milosevic, M.; Milosevic, D. (2013) "Integration of remote DSP experiments into moodle learning environment". In: The Fourth International Conference on e-Learning (eLearning 2013), p. 60-64.

Leite, W.S.S. (2014) "Sociedade moderna e tecnologias na educação: reflexões e perspectivas sobre a realidade no Brasil”. In: Revista Liberato, 2014, v. 15, n. 24.

Logar, V.; Karba, R.; Papić, Marko; Atanasijevic-Kunc, M. (2011) “Artificial and real laboratory environment in an e-learning competition". Journal of Mathematics and Computers in Simulation, 2011, v. 82, n. 3, p. 517-524.

Maciel, A.M.A.; Rodrigues, R.L.; Carvalho, E.C.B. (2014) "Desenvolvimento de um Assistente Virtual Integrado ao Moodle para Suporte a Aprendizagem Online". In: XXV Simpósio Brasileiro de Informática na Educação (SBIE 2014), p. 382-391. 
Mott, J. (2010) "Envisioning the Post-LMS Era: The Open Learning Network". In: Educause Quarterly, 2010, v. 33, n. 1, p. 1-9.

Mott, J.; Wiley, D. (2009) "Open for Learning: The CMS and the Open Learning Network". In: In education, 2009, v. 15, n. 2, 2013.

Ninoriya, S.; Chawan, P.M.; Meshram, B.B. (2011) "CMS, LMS and LCMS For eLearning”. In: International Journal of Computer Science (IJCSI 2011), v. 8, n. 2, p. 644-647.

Papić, M., Bešter, J. (2012) "Trends in ICT and Multimedia Supported Education". Journal Organizacija, vol. 45-3, 2012, pp. 131-139.

Park, J.Y., \& Mills, K.A. (2014). "Enhancing interdisciplinary learning with a learning management system". In: MERLOT Journal of Online Learning and Teaching, 2014, v. 10, n. 2 , p. $299-313$

Santana, M.A.; Neto, B.S.; Costa, E.B.; Silva, I.C.L. (2014) "Avaliando o Uso das Ferramentas Educacionais no Ambiente Virtual de Aprendizagem Moodle". In: XXV Simpósio Brasileiro de Informática na Educação (SBIE 2014), p. 278-287.

Siemens, G. (2005) "Connectivism: A learning theory for the digital age". In: International Journal of Instructional Technology and Distance Learning (IJITDL 2005), v. 2, n. 1, p. 3-10.

Silva, J.C.; Brito, A.V.; Mahon, F.; Medeiros, F.P.A. (2014) “Análise das interações dos alunos com Conteúdos e Atividades em Ambientes Colaborativos Virtuais de Aprendizagem". In: XXV Simpósio Brasileiro de Informática na Educação (SBIE 2014), p. 129-133.

Stone, D.E.; Zheng, G. "Handbook of Research on Education and Technology in a Changing Society". Information Science Reference: IGI Global, pp. 756-767.

Sun, J.C.Y. (2014) "Influence of polling technologies on student engagement: An analysis of student motivation, academic performance, and brainwave data". In: Computers \& Education, 2014, v. 72, p. 80-89.

Swacha, J.; Baszuro, P. (2013) "Gamification-based e-learning Platform for Computer Programming Education". In: X World Conference on Computers in Education (WCCE 2013), v. 1, p. 122-130.

Tarouco, L.M.R.; Schmitt, M.A.R.R.; Rodrigues, A.P.; Viccari, R.M. (2010) "Gestão colaborativa de conteúdo educacional”. In: RENOTE, 2010, v. 7, n. 1.

Wang, Y.; Chen, N. S. (2009) "Criteria for evaluating synchronous learning management systems: arguments from the distance language classroom". In: Computer Assisted Language Learning (CALL 2009), v. 22, n. 1, p. 1-18. 